Series A

I. MATHEMATICA

598

\title{
ON IRREDUCIBLE MODULES OF A LIE ALGEBRA WHICH ARE COMPOSED OF FINITE-DIMENSIONAL MODULES OF A SUBALGEBRA
}

BY

JOUKO MICKELSSON

H E L S I K I 1975

S U O M A L A I EN TIEDEAKATEMIA

doi:10.5186/aasfm.1975.598 
Copyright (C) 1975 by Academia Scientiarum Fennica

ISSN 0066-1953

ISBN 951-41-0214-2

Communicated 19 September 1974 


\section{Introduction}

Let $G$ be a Lie algebra and $K$ a subalgebra of $G$. If $K$ is semisimple (or at least reductive) then the finite-dimensional $K$-modules are well-known. We can then pose the following question: What are the irreducible $G$-modules which, when regarded as a $K$-module, are direct sums of irreducible finite-dimensional $K$-modules? We call such modules $K$-finite.

This problem has been extensively studied in the following special case (see e.g. [1]-[3], [5], [7]): Let $\mathscr{C}_{\text {g }}$ be a non-compact semisimple Lie group and let $\mathcal{K}$ be the maximal compact subgroup of $C_{\mathcal{L}}$. Let $G$ (resp. $K$ ) be the Lie algebra of $C_{1}$ (resp. K). As was shown by HarishChandra, study of unitary irreducible representations of $C_{l}$ in a Hilbert space leads in a natural way to a study of irreducible $K$-finite $G$-modules.

In this paper $G$ is an arbitrary (finite-dimensional) complex Lie algebra and $K$ is a semi-simple (or reductive) subalgebra of $G$. The work is divided into two parts. In section 3 we study irreducible $G$-modules admitting a vector of maximal weight $\lambda$ with respect to a Cartan subalgebra $H_{T}$ of $G$ such that $H=K \cap H_{T}$ is a Cartan subalgebra of $K$. We prove that for a sspecial» subalgebra $K$ (Definition 3.7) and for any weight $\lambda$ such that the restriction $\left.\lambda\right|_{H}$ is a dominant integral weight of $K$ there exists a unique equivalence class of $K$-finite $G$-modules which have the maximal weight $\lambda$.

In section 4 we study irreducible $G$-modules $V$ with the help of the minimal component $V_{\min }$ of $V$; if $\alpha$ is a dominant integral weight of $K$ we denote by $V_{x}$ the sum of all irreducible finite-dimensional $K$ modules in $V$ which have $\alpha$ as their maximal weight; by definition $V_{\min }=V_{\alpha}$ if $V_{\alpha} \neq 0$ and $V_{\beta}=0$ for all $\beta<x$. Let rank $G=\operatorname{rank} K$. We prove that if $\alpha$ is »large enough» (see Definition 4.1) then there exists a unique equivalence class $[V]$ of irreducible $K$-finite $G$-modules $V$ such that $V_{\min }=V_{\alpha}$. Such $G$-modules are called discrete because they are completely characterized by the weight $\alpha$ i.e. by a sequence of integers consisting of the components of $\alpha$.

We take profit at crucial steps (Theorem 3.9 and Lemma 4.7) of the results of J. Lepowsky and G. W. McCollum, [6]: If $V$ is a G-module 
such that $V_{\alpha} \neq 0$ for some dominant integral weight $\alpha$ and $V$ is generated by $V_{\alpha}\left(V=\mathscr{E}(C) V_{\alpha}\right.$ where $\mathscr{E}(G)$ is the enveloping algebra of $\left.G\right)$ then $V$ is $K$-finite. In addition $V$ is completely determined by the action of $\mathscr{E}(K) C$ on $V_{\alpha}$ where $C$ is the centralizer of $K$ in $\mathscr{E}(G)$. These results have earlier been obtained by Harish-Chandra, [1] and [2], in case $G$ is semi-simple.

See also for related recent results by van den Hombergh in "A note on Mickelsson's step algebra» and »On some Harish-Chandra modules» (to appear in Indagationes Mathematicæ).

The author would like to thank Professor Kalevi Suominen for reading the manuscript and suggesting improvements.

\section{Notation}

In this paper all Lie algebras are finite-dimensional. All algebras and vector spaces are over $\mathbf{C}$, the field of complex numbers. If $A$ is an algebra, $V$ an $A$-module, $S$ a subset of $A$ and $X$ a subset of $V$ we denote by $S X$ the subset $\{s x \mid s \in S, x \in X\}$ of $V$.

We denote by $V^{*}$ the dual space of a vector space $V$.

Let $K$ be a semi-simple Lie algebra, $H$ a Cartan subalgebra of $K$ and $\langle$,$\rangle the Killing form on K$. Because $\langle$,$\rangle is non-degenerate, there$ exists (for each $\alpha \in H^{*}$ ) an element $h_{\alpha} \in H$ such that $\left\langle h_{\alpha}, h\right\rangle=\alpha(h)$ for all $h \in H$. Let $($,$) be the symmetric non-degenerate bilinear form$ on $H^{*}$ defined by $(\alpha, \beta)=\left\langle h_{\alpha}, h_{\beta}\right\rangle$.

For any Lie algebra $G$, we denote by $x \mapsto$ ad $x$ the adjoint representation of $G, \operatorname{ad} x(y)=[x, y]$.

If $L$ is any Lie algebra then $\mathscr{E}(L)$ is the universal enveloping algebra of $L$. If $L^{\prime}$ is a subalgebra of $L$ then $\mathscr{E}\left(L^{\prime}\right)$ can be identified in a natural way with a subalgebra of $\mathscr{E}(L)$.

If $\left\{x_{1}, x_{2}, \ldots, x_{n}\right\}$ is a basis of $L$ then the monomials $x_{i(1)} x_{i(2)} \ldots x_{i(k)}$ $(i(1) \leqq i(2) \leqq \ldots \leqq i(k))$ along with 1 give a basis of $\mathscr{E}(L)$ (PoincaréBirkhoff-Witt theorem).

For any subset $S$ of $\mathscr{E}(L)$ we denote by ${ }^{\top}(S)$ the left ideal generated by $S, \mathscr{T}(S)=\mathscr{E}(L) S$.

Let $B$ be an associative algebra, $A_{1}$ an ideal of $B$ and $V$ a $B / A_{1}$ -module. If $\mathscr{A}_{2}$ is any ideal of $\mathcal{B}_{3}$ such that $\mathscr{A}_{2} \subset \mathscr{A}_{1}$ then by the extension of $V$ into a $B / \mathscr{A}_{2}$-module we mean the $B / \mathscr{A}_{2}$-module $V$, where the action of $B_{3} / A_{2}$ is defined by

$$
\left(b+\mathscr{A}_{2}\right) v=i(b) v ; b \in \mathscr{B}, v \in V,
$$

where $i: B_{3} \rightarrow B_{3} / \mathscr{A}_{1}$ is the canonical projection. 


\section{Modules with maximal weight}

If $L$ is any Lie algebra and $H_{L}$ a Cartan subalgebra of $L$ then we can write

$$
L=H_{L} \oplus \underset{\alpha \neq 0}{\oplus} L_{\alpha}
$$

where $\mathrm{L}_{\alpha}$ is the root subspace corresponding to the non-zero root $\alpha$. Because we assume $L$ to be finite-dimensional, the sum is finite. By definition $L_{\alpha}$ consists of all elements $x \in L$ such that

$$
(\operatorname{ad} h-\alpha(h))^{n} x=0 \text { for some positive integer } n \text {. }
$$

Let $H_{L}^{*}$ be the dual of $H_{L}$ and $\left\{h_{1}, h_{2}, \ldots, h_{l}\right\}$ a fixed basis of $H_{L}$. Let $\lambda, \mu \in H_{L}^{*}$ with $\lambda \neq \mu$. We say that $\lambda$ is bigger than $\mu(\lambda>\mu)$ if the first non-zero number in the sequence

$$
\lambda\left(h_{1}\right)-\mu\left(h_{1}\right), \ldots, \lambda\left(h_{l}\right)-\mu\left(h_{l}\right)
$$

is of the form $x+i y$ with $x>0$ or $x=0$ and $y>0$. We denote by $L_{+}\left(L_{-}\right)$the solvable subalgebra of $L$ generated by the subspaces $L_{\alpha}$ with $\alpha>0(\alpha<0)$.

Definition 3.1. Let $V$ be an $L$-module. We denote by $V^{+}$the subspace of $V$ consisting of all vectors $v$ with the property $x v=0$ for all $x \in L_{+}$. We say that $V$ is bounded above if $1 \leqq \operatorname{dim} V^{+}<\infty$.

Lemma 3.2. If $V$ is an irreducible $L$-module which is bounded above then $\operatorname{dim} V^{+}=1$ and there exists $\lambda \in H_{L}^{*}$ such that $h v=\lambda(h) v$ for each $v \in V^{+}$and $h \in H_{L}$. (We say that $\lambda$ is the maximal weight of $V$ and $v$ is a maximal vector.)

Proof. Because $1 \leqq \operatorname{dim} V^{+}<\infty$ and $H_{L}$ is nilpotent there is a common eigenvector $v \in V^{+}$of all $h \in H_{L}$ ( $V^{+}$is clearly $H_{L}$-invariant). Because of the irreducibility of $V$ and of the Poincaré-Birkhoff-Witt theorem we have $V=\mathscr{E}\left(L_{-}\right) v$. If $v^{\prime}=u v, u \in \mathscr{E}\left(L_{-}\right) L_{-}$, is another vector in $V^{+}$then

$$
V^{\prime}=\mathscr{E}(L) v^{\prime}=\mathscr{E}\left(L_{-}\right)^{\mathscr{E}}\left(H_{L}\right) v^{\prime}
$$

is a non-trivial $\left(v \notin V^{\prime}\right)$ invariant subspace if $v^{\prime} \neq 0$. Thus $v^{\prime}=0$ and $\{v\}$ is a basis of $V^{+}$.

Definition 3.3. Let $V$ be an $L$-module. For each $\alpha \in H_{L}^{*}$ the weight subspace $V_{\alpha}$ consists of all vectors $v \in V$ for which

$(h-\alpha(h))^{n} v=0$ for all $h \in H_{L}$ and for some positive integer $n$.

Theorem 3.4. Let $L$ be a Lie algebra and let $H_{L}$ be a Cartan subalgebra of $L$. For each $\lambda \in H_{L}^{*}$ such that $\lambda_{\left[H_{L}, H_{L}\right]}=0$ there exists a unique equivalence class of irreducible $L$-modules which are bounded above and have maximal weight $\lambda$. Any such an L-module is a direct sum of weight subspaces 
of finite dimension.

Proof. We define $W^{\lambda}=\mathscr{E}(L) / q_{\lambda}$ where $g_{\lambda}$ is an ideal,

$$
\rho_{\lambda}=9\left(L_{+}\right)+9\left(\left\{h-\lambda(h) \cdot \mathbf{1} \mid h \in H_{L}\right\}\right) \text {. }
$$

$W^{i}$ is an $L_{-}$(and $\mathscr{E}(L)-$ ) module in a natural way. Let $v_{0}=\mathbf{1}+g_{i}$. Then

$$
L_{+} v_{0}=0, h v_{0}=\lambda(h) v_{0} \quad\left(h \in H_{L}\right), W^{\lambda}=\mathscr{E}\left(L_{-}\right) v_{0} .
$$

It follows that a basis of $W^{\lambda}$ is given by vectors of the type

$$
e_{\beta_{1}} e_{\beta_{2}} \ldots e_{\beta_{k}} v_{0}(k=0,1,2, \ldots)
$$

where $e_{\beta_{i}}(i=1,2, \ldots, k)$ is any element of some fixed basis of $L_{\beta_{i}}$ and $0>\beta_{1} \geqq \beta_{2} \geqq \ldots \geqq \beta_{k}$ are negative roots of $L$. Let $W_{\alpha}^{\lambda}$ be the subspace of $W^{\lambda}$ spanned by the vectors $\left(^{*}\right)$ for which

$$
\alpha=\beta_{1}+\ldots+\beta_{k}+\lambda .
$$

We show by induction on $k$ than $W_{\alpha}^{\lambda}$ is a weight subspace with weight $\alpha$. Assume that the vector $v$ has weight $\beta$,

$$
(h-\beta(h))^{n} v=0 \text { for all } h \in H_{L} .
$$

Let $\gamma$ be a root and $e_{\gamma} \in L_{\gamma}$,

$$
(\text { ad } h-\gamma(h))^{m} e_{\gamma}=0 \text { for all } h \in H_{L} .
$$

Then

$$
\begin{aligned}
& (h-(\beta+\gamma)(h))^{n+m} e_{\gamma} v=\sum_{k=0}^{n+m}(\operatorname{ad} h-\gamma(h))^{k} e_{\gamma} \cdot\left(\begin{array}{c}
n+m \\
k
\end{array}\right) . \\
& \cdot(h-\beta(h))^{n+m-k} v=0 .
\end{aligned}
$$

Thus the we ght of $e_{\gamma} v$ is $\beta+\gamma$. It follows that each $v \in W_{\alpha}^{i}$ is of weight $\alpha \leqq \lambda$. It is clear that $\operatorname{dim} W_{\alpha}^{i}<\infty$ and each vector of weight $\alpha$ belongs to $W_{\alpha}^{i}$. Note that $W_{i}^{i}$ is spanned by the vector $v_{0}=\mathbf{1}+?_{i}$. Let $N^{\lambda}$ be the sum of all invariant subspaces in $W^{i}$ which do not contain $v_{0}$. Then $v_{0} \notin N^{\lambda}$ and we define

$$
V^{i}=W^{i} / \Lambda^{i} .
$$

The $L$-module $V^{\lambda}$ is irreducible, has $v_{0}+N^{i}$ as the maximal vector and $\lambda$ is the maximal weight. The uniqueness part of the proof goes as in the case of a semisimple Lie algebra (see [4, p. 109]).

Let $G$ be a Lie algebra, $K$ a semi-simple subalgebra of $G$ and $H$ a Cartan subalgebra of $K$. Because $K$ is semi-simple, there exists a subspace $T$ in $G$ such that $G=K \oplus T$ and $[K, T] \subset T$. We denote by $T_{0}$ the null component of $H$ in $T$, 


$$
T_{0}=\{x \in T \mid[h, x]=0 \quad \forall h \in H\} .
$$

Lemma 3.5. Let $H_{T}$ be a Cartan subalgebra of the Lie algebra $H+$ $T_{0} \subset G$. Then $H \subset H_{T}$ and $H_{T}$ is even a Cartan subalgebra of $G$.

Proof. There exists $x \in H+T_{0}$ such that

$$
H_{T}=\left\{y \in H+T_{0} \mid(\operatorname{ad} x)^{n} y=0 \text { for some } n \in N\right\},
$$

$\left[4\right.$, pp.79-80]. Now $[x, H]=0$ for all $x \in H+T_{0}$, thus $H \subset H_{T}$. Next let $S$ be the normalizer of $H_{T}$ in $G$. From $\left[S, H_{T}\right] \subset H_{T}$ it follows that $[S, H]=0$ and therefore $S \subset H+T_{0}$. Because $H_{T}$ is a Cartan subalgebra of $H+T_{0}$ it follows that $S=H_{T}$ and we can conclude that $H_{T}$ is a Cartan subalgebra of $G$.

Let $\Phi$ be the set of roots of $K$ relative to $H, \Delta \subset \Phi$ is a set of simple roots and $\Phi^{+}$(resp. $\Phi^{-}$) is the set of positive (resp. negative) roots with respect to $\Delta$. Next we divide $T$ into weight subspaces,

$$
T_{\lambda}=\{x \in T \mid[h, x]=\lambda(h) x, \mathrm{~V} h \in H\} .
$$

We denote by $\Psi$ the set of weights of $K$ in $T, \Psi^{+}$(resp. $\Psi^{-}$) is the set of positive (resp. negative weights in $\Psi$ relative to an ordered basis $\left\{h_{1}, \ldots, h_{l}\right\}$ of $H$ which is dual to the basis $\left\{h_{\alpha_{1}}, \ldots, h_{\alpha_{l}}\right\}$,

$$
\alpha_{i}\left(h_{j}\right)=\left\langle h_{\alpha_{i}}, h_{j}\right\rangle=\delta_{i j} .
$$

Here $\alpha_{1}, \ldots, \alpha_{l}$ are the distinct simple roots of $K$.

Definition 3.6. The semi-simple subalgebra $K$ of $G$ is a special subalgebra if

$$
\mathbf{N}(\{\alpha\}) \cap \mathbf{N}\left(\Psi^{+}\right)=\{0\}
$$

for all $\alpha \in \Delta$. If $\Omega \subset H^{*}$ is any subset, we denote by $\mathbf{N}(\Omega)$ the linear span of $\Omega$ with non-negative integral coefficients.

Example 3.7. Let $G=\operatorname{gl}(n, \mathbf{C})$, the Lie algebra with basis $\left\{e_{i j}\right\}_{i, j=1}^{n}$ and commutation relations

$$
\left[e_{i j}, e_{k l}\right]=\delta_{j k} e_{i l}-\delta_{i l} e_{k j} .
$$

Let $K$ be the subalgebra spanned by the vectors $(2 \leqq p \leqq n-2)$

$$
e_{i j}, \quad i \neq j, \quad 1 \leqq i, j \leqq p ; e_{i i}-e_{i-1 i \div 1}, i=1,2, \ldots, p-1 ;
$$

and

$e_{i j}, i \neq j, p+1 \leqq i, j \leqq n ; e_{i i}-e_{i+1 i+1}, i=p+1, p+2, \ldots, n-1$.

Note that $K$ is isomorphic to $A_{p-1} \oplus A_{n-p-1}$. As $H$ we can take the subalgebra of $K$ spanned by the vectors $e_{i i}-e_{i+1 i+1}, 1 \leqq i \leqq n-1, i \neq p$. It is easily seen that in this case 


$$
T_{0}=\left\{a \sum_{i=1}^{p} e_{i i}+b \sum_{i=p+1}^{n} e_{i i} \mid a, b \in \mathbf{C}\right\}
$$

and $H_{T}=H+T_{0}$. Using the properties of the roots of the classical simple Lie algebras $A_{l}$ it is not difficult to verify that $K$ is special.

We return to the general case. Let $K$ be a special subalgebra of $G$ and $l=\operatorname{rank} K, p=\operatorname{rank} G$. We fix an ordered basis $\left\{h_{1}, h_{2}, \ldots, h_{p}\right\}$ of $H_{T}$ such that $\left\{h_{1}, h_{2}, \ldots, h_{l}\right\}$ is the basis of $H$ described above. We define the following subalgebras of $G$ :

$$
\begin{aligned}
& G_{+}=K_{+}+\sum_{\lambda>0} T_{\lambda}+S_{+}, \\
& G_{-}=K_{-}+\sum_{\lambda<0} T_{\lambda}+S_{-}
\end{aligned}
$$

where $K_{+}$(resp. $K_{-}$) is the subalgebra of $K$ spanned by the vectors belonging to positive (resp. negative) roots of $K$. We define

$$
H+T_{0}=S_{+}+S_{-}+H_{T}
$$

to be the corresponding decomposition for $H+T_{0}$. Because of our choice of basis of $H_{T}$ (see also Lemma 3.5) it is clear that $G=G_{+}+G_{-}+H_{T}$ is a similar decomposition for $G$ relative to the Cartan subalgebra $H_{T}$.

Definition 3.8. A $G$-module $V$ is $K$-finite if it is a sum of finitedimensional $K$-modules when considered as a $K$-module by restriction to $K$.

Let $\Lambda$ be the set of dominant integral elements in $H^{*}$ :

$$
\Lambda=\left\{\lambda \in H^{*} \mid(\lambda, \alpha) \text { is a non-negative integer for all } \alpha \in \Delta\right\} .
$$

Theorem 3.9. Let $K$ be a special subalgebra of $G$. Then for each $\lambda \in H_{T}^{*}$ such that $\left.\lambda\right|_{H} \in \Lambda$ and $\left.\lambda\right|_{\left[H_{T}, H_{T}\right]}=0$ there exists a unique equivalence class of $K$-finite irreducible $G$-modules which are bounded above and have $\lambda$ as the maximal weight.

Proof. The uniqueness follows from Theorem 3.4. We have to prove the existence. We define an ideal

$$
\tau_{\lambda}=9\left(G_{+}\right)+\vartheta\left(\left\{h-\lambda(h) \cdot \mathbf{1} \mid h \in H_{T}\right\}\right)
$$

and $W^{\lambda}=\mathscr{E}(G) / q_{\lambda}$. Consider the subset $S_{\lambda}$ of $W^{\lambda}$,

$$
S_{\lambda}=\left\{e_{-\alpha}^{n_{\alpha}+1}+\varphi_{\lambda} \mid \alpha \in \Delta\right\}
$$

where $e_{-\alpha}$ belongs to the root $-\alpha$ and

$$
n_{\alpha}=2 \cdot \frac{\left(\left.\lambda\right|_{H}, \alpha\right)}{(\alpha, \alpha)}, \alpha \in \Delta .
$$

Let $U^{\lambda}=\mathscr{E}(G) S_{\lambda}$ be the submodule of $W^{\lambda}$ generated by $S_{\lambda}$. We claim that $U^{\lambda}$ does not contain the vector $\mathbf{1}+9_{\lambda}$. It is well-known that $S_{\lambda}$ 
is annihilated by $K_{+}$(see [4, p. 115]). Now $W^{\lambda}$ is a direct sum of weight subspaces, $W_{\lambda}^{\lambda}$ is spanned by the vector $1+9_{\lambda}$ and $\lambda$ is the highest weight in $W^{\lambda}$ (compare the proof of Theorem 3.4). Suppose that $1+$ $q_{\lambda} \in U^{\lambda}$; using the Poincaré-Birkhoff-Witt theorem it is easily seen that then there exists $\beta_{1}, \beta_{2}, \ldots, \beta_{k} \in \Psi^{+}$such that

$$
\beta_{1}+\beta_{2}+\ldots+\beta_{k}-\left(n_{\alpha}+1\right) \cdot \alpha=0
$$

for at least one weight $\alpha \in \Delta$. But this is impossible because $K$ is a special subalgebra of $G$.

Let again $N^{\lambda}$ be the sum of all invariant subspaces of $W^{\lambda}$ not containing the vector $1+9_{\lambda}$. It is clear that $U^{i} \subset N^{i}$. We define

$$
V^{i}=W^{i} / N^{i} \text {. }
$$

The $G$-module $V^{i}$ is irreducible and has a maximal vector $v=1+y_{2}+$ $N^{\lambda}$ of weight $\lambda$. Furthermore, $V^{\lambda}$ contains a finite-dimensional $K$-module, namely $\mathscr{E}(K) v([4, \mathrm{p} .115])$. It follows from proposition 4.2 , [6], that $V^{2}$ is $K$-finite. (See also [1, Theorem 1.])

\section{Discrete G-modules}

If not otherwise stated, the notation of the previous sections is in force also in this section.

Let $C$ be the centralizer of $K$ in $\mathscr{E}(G)$. The algebra $C$ is a finitely generated subalgebra of $\mathscr{E}(G)$ (see [8, p. 162, Theorem 2.3.1.4]).

Let an ordered basis $\left\{t_{1}, t_{2}, \ldots, t_{r}\right\}$ be given for the subspace $T$ of $G$, such that

$$
\left[h, t_{i}\right]=\lambda_{i}(h) t_{i}, h \in H, i=1,2, \ldots, r,
$$

where $\lambda_{i} \in \Psi \quad(i=1,2, \ldots, v)$ and $\lambda_{1} \geqq \lambda_{2} \geqq \ldots \geqq \lambda_{r}$. We complete this to an ordered basis of $G$,

$$
\left\{t_{i}, e_{\alpha_{i}}, h_{i}, e_{\beta_{i}}\right\}
$$

where the order is defined through the ordering of roots,

$$
\alpha_{1}<\alpha_{2}<\ldots<\alpha_{q}<0<\beta_{1}<\beta_{2}<\ldots<\beta_{q},
$$

and through a labelling of the basis elements $h_{i}$ of $H$. According to the Poincaré-Birkhoff-Witt theorem this ordering induces a basis for $\mathscr{E}(G)$ by ordered monomials in the basis elements of $G$. If $u \in \mathcal{E}(G)$ is such a basis vector we denote by $\operatorname{deg}(u)$ the number of vectors $t_{i}$ contained in $u$. If $v \in \mathscr{E}(G)$ is an arbitrary (finite) linear combination of ordered monomials, 


$$
v=\sum_{k=1}^{n} a_{k} u_{k}\left(a_{k} \in \mathbf{C}\right)
$$

we define $\operatorname{deg}(v)=\max _{k=1, \ldots, n} \operatorname{deg}\left(u_{k}\right)$.

Definition 4.1. Let $c_{1}, c_{2}, \ldots, c_{\zeta}$ be a generating sequence of $C$. We define

$$
n_{c}=\max _{k=1,2, \ldots, \zeta} \operatorname{deg}\left(c_{k}\right) .
$$

We call an element $\lambda$ of $\Lambda$, the set of dominant integral weights of $K$, large if

$$
\lambda+\omega_{1}+\omega_{2}+\ldots+\omega_{k} \in \Lambda \text { for all } \omega_{i} \in \Psi, k=1,2, \ldots, n_{c} .
$$

Let $V$ be any $G$-module. Consider $V$ as a $K$-module by restriction. For any $\lambda \in A$ we denote by $V_{\lambda}$ the sum of all irreducible finite-dimensional $K$-submodules of $V$ with maximal weight $\lambda$. We define

$$
V_{i}^{+}=\left\{x \in V_{\lambda} \mid e_{\beta} x=0 \quad \mathrm{~V} \beta \in \Phi^{+}\right\},
$$

the subspace of vectors with maximal weight in $V_{j}$; in other words,

$$
V_{i}^{+}=\left\{x \in V_{i} \mid h x=\lambda(h) x \quad \forall h \in H\right\} .
$$

We denote by $g_{\lambda}$ the annihilator in $\mathscr{E}(K)$ of the maximal vector in an irreducible finite-dimensional $K$-module with maximal weight $\lambda$; according to $[4$, p. 115],

$$
g_{\lambda}=9\left(K_{+}\right)+9(\{h-\lambda(h) \cdot 1 \mid h \in H\})+9\left(\left\{e_{-\alpha}^{n_{\alpha}+1} \mid \alpha \in \Delta\right\}\right)
$$

where $K_{+}$and the numbers $n_{\alpha}$ are defined as in section 3 .

For all $\beta$ and $\alpha$ in $\Lambda$ we define $A_{\beta, \alpha}$ to be the subset of $\mathscr{E}(G)$ for which

$$
A_{\beta, \alpha} V_{\alpha}^{+} \subset V_{\beta}^{+}
$$

for any $G$-module $V$.

Lemma 4.2. $A_{\beta, \alpha}=\left\{u \in \mathscr{E}(G) \mid g_{\beta} u \subset \mathscr{E}(G) g_{\alpha}\right\}$.

Proof. Let $V$ be a $G$-module such that $V_{x} \neq 0$. Any such $G$-module is a factor module of the left-module $\mathscr{E}(G) / \mathscr{E}(G) q_{\alpha}$. It follows that

$$
A_{\beta, \alpha}=\left\{u \in \mathscr{E}(G) \mid u V_{\alpha}^{+} \subset V_{\beta}^{+}\right\}
$$

where $V=\mathscr{E}(G) / \mathscr{E}(G) g_{\alpha}$. Let now $u \in A_{\beta, \alpha}$. If $x=1+\mathscr{E}(G) g_{\alpha}$ then $x \in V_{\alpha}^{+}$and

$$
u x=u+\mathscr{E}(G){ }_{\alpha}^{9} \in V_{\beta}^{+}
$$

and therefore ${ }_{\beta} u \subset \mathscr{E}(G){ }_{\alpha}$. To prove the converse, assume that $g_{\beta} u \subset \mathscr{E}(G) g_{\alpha}$. Let $x \in V_{\alpha}^{+}$. Then 


$$
g_{\beta} u x \subset \mathscr{E}(G) g_{x}=0 .
$$

It follows that $\mathscr{E}(K) u x$ is a finite-dimensional $K$-module with $u x$ as the vector of maximal weight (which is $\beta$ ) and thus $u x \in V_{\beta}^{+}$.

Lemma 4.3. Let $\omega$ be an element of $\Lambda$ such that $\omega+\lambda \in \Lambda$ for any $\lambda \in \Psi$. Then for each $t_{i} \in T$ there exists $u \in A_{\omega+i_{i}, \omega}$ of the form

$$
u=t_{i}+\sum_{j, i_{j}>\lambda_{i}} t_{j} v_{j}
$$

where $v_{j} \in \mathscr{E}\left(K_{-}\right)$.

Proof. We can write

$$
T=\underset{v}{\oplus} T^{(v)}
$$

where $T^{(v)}$ is the irreducible component of $T$ under the adjoint action of $K$, with maximal weight $v$. We can assume that the basis $\left\{t_{j}\right\}_{j=1}^{r}$ of $T$ is chosen in such a way that it is compatible with the decomposition (*); thus we may assume that $t_{i} \in T^{(v)}$ for some weight $v$.

Put $D_{\omega}=\mathscr{E}(K) / g_{\omega}$ and consider the tensor product $T^{(v)} \otimes D_{\omega}$, which is a $K$-module under the diagonal action:

$$
k(x \otimes y)=[k, x] \otimes y+x \otimes k y ; k \in K, x \in T^{(v)}, y \in D_{\omega} .
$$

It is known that the module $T^{(v)} \otimes D_{\omega}$ contains an irreducible submodule with maximal weight $\omega+\lambda_{i}$ (note that $\omega+\lambda \in \Lambda$ for any weight $\lambda$ in $T^{(v)}$ ) with a multiplicity which is equal to the multiplicity $m\left(\lambda_{i}, v\right)$ of the weigth $\lambda_{i}$ in $T^{(v)}$; in other words there are $m\left(\lambda_{i}, v\right)$ linearly independent vectors in $T^{(v)} \otimes D_{(1)}$ which are annihilated by $g_{(1)+\hat{\lambda}_{i}}$ (see e.g. [4, pp. 141-142]). It follows that for each $t_{i} \in T_{i_{i}}^{(v)}=T_{\lambda_{i}} \cap T^{(v)}$ there exists a nonzero element $u_{0}$ of $T^{(v)} \otimes D_{(1)}$ of the form

$$
\begin{gathered}
u_{0}=t_{i} \otimes\left(a \cdot 1+g_{\omega}\right)+\sum_{\substack{j \\
i_{j}>i_{i}}} t_{j} \otimes\left(v_{j}+g_{\omega}\right), \\
a \in \mathbf{C}, \quad v_{j} \in \mathscr{E}\left(K_{-}\right),
\end{gathered}
$$

such that $u_{0}$ is annihilated by $g_{\omega+\lambda_{i}}$. We define $v_{i}=a \cdot 1$ and let $k$ be the smallest value of the index $j$ for which $v_{j} \notin g_{\omega}$; because of

$$
e_{\alpha} u_{0}=0 \text { for all } \alpha \in \Phi^{+},
$$

we have $e_{\alpha} v_{k} \in 9_{(1)}$ for all $\alpha \in \Phi^{+}$. Now any vector in $D_{\omega}$ which is annihilated by $K_{+}$is a multiple of $1+9_{\omega}$; thus $k=i$ and $a \neq 0$. We may assume that $a=1$ (multiply $u_{0}$ by $a^{-1}$ ).

Consider the linear mapping 


$$
\varphi: T^{(v)} \otimes D_{\omega} \rightarrow \mathscr{E}(G) / \mathscr{E}(G) \mathscr{g}_{\omega}
$$

induced by the multiplication map $T^{(v)} \otimes \mathscr{E}(K) \rightarrow \mathscr{E}(G)$. This mapping is a $K$-module homomcrphism; in fact,

$$
\begin{gathered}
\varphi\left(k\left(t \otimes\left(v+g_{\omega}\right)\right)\right)=\varphi\left([k, t] \otimes\left(v+g_{\omega}\right)+t \otimes\left(k v+g_{\omega}\right)\right) \\
=[k, t] v+\mathscr{E}(G) g_{\omega}+t k v+\mathscr{E}(G) g_{\omega}=k t v+\mathscr{E}(G) g_{\omega} \\
=k \varphi\left(t \otimes\left(v+g_{\omega}\right)\right),
\end{gathered}
$$

for all $k \in K, t \in T^{(v)}$ and $v \in \mathscr{E}(K)$. Let $u \in \mathscr{E}(G)$,

$$
u=t_{i}+\sum_{j, i_{j}>\lambda_{i}} t_{j} v_{j} .
$$

Then $\left.\varphi\left(u_{0}\right)\right)=u+\mathscr{E}(G) g_{\omega}$ and therefore $g_{\omega+i_{i}} u \subset \mathscr{E}(G) g_{\omega}$. In other words (Lemma 4.2), $u \in A_{\omega+i_{i},(\omega)}$.

We denote by $P$ the projection $P: \mathscr{E}(G) \rightarrow{ }^{\mathscr{E}}(G)$ such that Ker $P=$ $\mathscr{E}(G)_{\alpha}+U_{1}^{\mathscr{E}}\left(K_{-}\right)$and $P(\mathscr{E}(G))=U_{1}$ where $U_{1}$ consists of the elements

$$
b \cdot 1+\sum a_{i_{1} \ldots i_{k}} t_{i_{1}} \ldots t_{i_{k}}
$$

where $b, a_{i_{1} \ldots i_{k}} \in \mathbf{C}$ and $i_{1} \leqq \ldots \leqq i_{k}$.

Lemma 4.4. Let $u_{1}, u_{2} \in A_{\beta, \alpha}$ such that $P\left(u_{1}\right)=P\left(u_{2}\right)$. Then

$$
u_{1}-u_{2} \in \mathscr{E}(G) g_{\alpha} .
$$

Proof. We shall again use the fact that any vector in $\mathscr{\&}(K) \overbrace{\alpha}$ which is annihilated by $K_{+}$is a multiple of $1+9_{\alpha}$. First we write

$$
u_{1}-u_{2}=w+t_{j_{1}} \ldots t_{j_{k}} v+\sum_{\left\{m, i_{v}\right\}} t_{i_{1}} \ldots t_{i_{m}} v_{i_{1} \ldots i_{m}}
$$

where each term is a sum of ordered monomials and $w \in \mathscr{E}(G) g_{\omega}, v$ and $v_{i_{1} \ldots i_{m}} \in \mathscr{E}\left(K_{-}\right)$and

$$
\lambda_{i_{1}}+\ldots+\lambda_{i_{m}} \geqq \lambda_{j_{1}}+\ldots+\lambda_{j_{k}} .
$$

If $m=k$ then $j_{v} \neq i_{v}$ for at least one value of the index $v$. From $K_{+}\left(u_{1}-u_{2}\right) \in \mathscr{E}(G) g_{\alpha}$ it follows that

$$
K_{+} v \subset 9_{\alpha}
$$

and thus $v \in g_{\alpha}\left(v \notin a \cdot 1+g_{\alpha}\right.$ for any $a \neq 0$ because of $P\left(u_{1}-u_{2}\right)$ $=0)$. By induction it follows that the coefficient of any $t_{i_{1}} \ldots t_{i_{m}}$ belongs to $g_{\alpha}$ and therefore $u_{1}-u_{2} \in \mathscr{E}(G) ?_{\alpha}$.

It is clear that Lemma 4.4 is valid also if we replace $A_{\beta, \alpha}$ by

$$
A_{\alpha}=\sum_{\beta} A_{\beta, \alpha}
$$


Lemma 4.5. Let $\alpha \in \Lambda$ be large. Then any $u \in A_{\alpha}$ such that $\operatorname{deg}(u) \leqq n_{c}$ can be written in the form

$$
\begin{gathered}
u=v+a \cdot \mathbf{1}+\sum_{k,\left\{i_{v}\right\}} u_{i_{1}} u_{i_{2}} \ldots u_{i_{k}} \quad\left(a \in C, \quad v \in \mathscr{E}(G) g_{\alpha}, \quad u_{i_{\nu}} \in \mathscr{E}(G) ;\right. \\
v=1,2, \ldots, k)
\end{gathered}
$$

where $k \leqq \operatorname{deg}(u), i_{1} \leqq i_{2} \leqq \ldots \leqq i_{k}$ and

$\left(^{*}\right) \quad u_{i_{v}} u_{i_{v+1}} \ldots u_{i_{k}} \in A_{\delta_{v}, \alpha} ; \delta_{v}=\alpha+\lambda_{i_{v}}+\ldots+\lambda_{i_{k}}(v=1,2, \ldots, k)$.

Proof. (1) Let $S$ be the set consisting of finite sequences $\boldsymbol{i}=$ $\left(i_{1}, i_{2}, \ldots, i_{k}\right)$ where $k \leqq n_{c}$ and the integers $i_{v}$ satisfy the inequalities

$$
0<i_{1} \leqq i_{2} \leqq \ldots \leqq i_{k} \leqq r=\operatorname{dim} T \text {. }
$$

We denote by $\boldsymbol{e}$ the empty sequence. We define an order in $S$ by putting

$$
\left(i_{1}, i_{2}, \ldots, i_{k}\right)<\left(j_{1}, j_{2}, \ldots, j_{m}\right) \text { if } k<m \text { or } k=m \text { and }
$$

the first non-zero number in the sequence $i_{1}-j_{1}, i_{2}-j_{2}, \ldots$ is positive. In addition, for each $i \in S$ we define

$$
t_{i}=t_{i_{1}} \ldots t_{i_{k}} \in \mathscr{E}(G)
$$

and $t_{\boldsymbol{e}}=1 \in \mathscr{E}(G)$. Let $V$ be the subspace of $\mathscr{E}(G)$ which has the set $\left\{t_{i} \mid \boldsymbol{i} \in S\right\}$ as an ordered basis (the order is defined through the ordering of $S)$.

(2) We put $u^{e}=1 \in A_{\alpha}$. From the fact that $\alpha$ is large and from Lemma 4.3. it follows that for each $\boldsymbol{i}=\left(i_{1}, i_{2}, \ldots, i_{k}\right) \in S$ there exists

$$
u^{i}=u_{1}^{i} u_{2}^{i} \ldots u_{k}^{i} \in A_{\alpha}\left(u_{\nu}^{i} \in \mathcal{E}(G) ; v=1,2, \ldots, k\right)
$$

where each $u_{v}^{i}$ is of the type described in Lemma $4.3, P\left(u_{v}^{i}\right)=t_{i_{v}}$ and $u_{\mathbf{i}}$ satisfies the relations $\left({ }^{*}\right)$. We denote by $U$ the subspace of $A_{\alpha}$ which has the set

$$
\left\{u^{i} \mid i \in S\right\}
$$

as an ordered basis.

(3) It is clear that the operator $P$ induces a linear mapping from $U$ into $V$. Furthermore,

$$
P\left(u^{i}\right)=t_{i}+\text { lower terms }
$$

as follows easily from the properties of the $u^{i}: s$ (see Lemma 4.3). Thus the matrix representing $P$ is triangular in the ordered basis described above, the diagonal elements being equal to 1 . It follows that the inverse of $P$ exists and therefore for each $u \in A_{\alpha}$, deg $(u) \leqq n_{c}$, there exists $u^{\prime} \in U$ such that 


$$
P(u)=P\left(u^{\prime}\right)
$$

(Note that $P(u) \in V$.) From Lemma 4.4 it follows that there exists $v \in \mathscr{E}(G) \mathscr{S}_{\alpha}$ such that $u=v+u^{\prime}$.

Lemma 4.6. Let $\alpha, \beta \in A$, and let $V$ be an irreducible G-module such that $V_{\alpha} \neq 0 ;$ then $V_{\beta}^{+}=A_{\beta, \alpha} V_{\alpha}^{+}$.

Proof. It is sufficient to prove the statement for $V=\mathscr{E}(G) / \mathscr{E}(G) g_{\alpha}$ (compare with the proof of Lemma 4.2). Then

$$
\begin{aligned}
V_{\beta}^{+} & =\left\{u+\mathscr{E}(G) g_{\alpha} \mid g_{\alpha} u \subset \mathscr{E}(G) g_{\alpha}\right\} \\
& =A_{\beta, \alpha}+\mathscr{E}(G) g_{\alpha}=A_{\beta, \alpha}\left(1+\mathscr{E}(G) g_{\alpha}\right) \\
& \subset A_{\beta, \alpha} V_{\alpha}^{+} .
\end{aligned}
$$

The relation $A_{\beta, \alpha} V_{\alpha}^{+} \subset V_{\beta}^{+}$follows from the definition of $A_{\beta, \alpha}$.

Let $C$ be the centralizer of $K$ in $\mathscr{\&}(G)$. If $V$ is any $G$-module then $V_{\alpha}$ and $V_{\alpha}^{+}$are $C$-modules by restriction of $\mathscr{E}(G)$ to the subalgebra $C$; in fact $V_{\alpha}$ is even a $\mathscr{E}(K) C$-module.

Lemma 4.7. Let $V$ be an irreducible G-module, $V_{\alpha} \neq 0$. Then the equivalence class $[V]$ of $V$ is completely determined by the equivalence class of the C-module $V_{\alpha}^{+} . V_{\alpha}^{+}$is an irreducible $C$-module.

Proof. This is an easy consequence of Theorem 5.5, [6]. (Note that the action of $\mathscr{E}(K) C$ on $V_{\alpha}$ is completely determined by the action of $C$ on $V_{\alpha}^{+}$.)

Let $G_{\alpha}^{\prime}$ be the set of all equivalence classes $[V]$ of irreducible $G$ modules $V$ such that $V_{\alpha} \neq 0$ and $V_{\alpha}=0$ for each $\beta<\alpha$. We call $V_{\alpha}$ the minimal component of $V$. Now an irreducible $G$-module $V$ is $K$-finite if and only if $V_{x} \neq 0$ for some weight $x \in A, \quad[6$, proposition 4.2]. It follows that $V$ is $K$-finite if and only if $V$ has a minimal component. Thus the set $G^{\prime}$ of all equivalence classes of irreducible $K$-finite $G$ modules is equal to

$$
\bigcup_{\alpha \in \Lambda} G_{\alpha}^{\prime}
$$

Of course $G_{\alpha}^{\prime} \cap G_{\beta}^{\prime}=\phi$ when $\alpha \neq \beta$.

Let $M_{\alpha}=\sum_{\beta<\alpha} A_{\beta, \alpha}$. If $[V] \in G_{\alpha}^{\prime}$ then $V_{\alpha}^{+}$is in a natural way a $C / C \cap \mathscr{E}(G) M_{\alpha}$-module. We denote by $C_{\alpha}^{\prime}$ the set of all equivalence classes of irreducible $C / C \cap \mathscr{E}(G) M_{\alpha}$-modules.

Theorem 4.8. The mapping $V \rightarrow V_{\alpha}^{+}$induces a bijection between $G_{\alpha}^{\prime}$ and $C_{\alpha}^{\prime}$.

Proof. If $[V],[W] \in G_{\alpha}^{\prime}$ then it is clear that $V_{\alpha}^{+}$and $W_{\alpha}^{+}$are equivalent as $C$-modules if and only if they are equivalent as $C / C \cap \mathscr{E}(G) M_{\alpha^{-}}$ modules. The injectivity of the mapping follows now from Lemma 4.7.

Let next $[W] \in C_{\alpha}^{\prime}$. We have to show that there exists $[V] \in G_{\alpha}^{\prime}$ such that $V_{\alpha}^{+} \cong W$ as $C / C \cap \mathscr{E}(G) M_{\alpha}$-modules. First we extend $W$ to a $\mathrm{C}$ - 
module. Let $x$ be a non-zero element of $W$, and let $\mathscr{L}$ be the annihilator of $x$ in $C$ so that $W=C / \mathscr{L}$. We define a left ideal of $\mathscr{E}(G)$ by

$$
\eta=\left\{\left.u \in \mathscr{E}(G)\right|^{\mathscr{E}}(G) u \cap C \subset \mathscr{L}\right\} .
$$

Consider the $G$-module $V=\mathscr{E}(G) / \nearrow$. First we show that $V$ is irreducible i.e. the left ideal $\nearrow$ is maximal. Let $q n \subset \mathcal{E}(G)$ be a left ideal such that $1 \notin 9 n$ and $~ n \subset m$. Then

$$
\mathscr{L}=C \cap 92 \subset C \cap \mathscr{E}(G) \mathrm{m} \text {. }
$$

Because of the irreducibility of $W, \mathscr{L}$ is a maximal left ideal in $C$. Now $1 \notin C \cap \mathscr{E}(G) M$ and therefore $\mathscr{L}=C \cap \mathscr{E}(G) 9 m$. From the definition of $M$ it follows that $m \subset 9$; thus $9 m=9$ and 92 is maximal.

Since $g_{\alpha} \subset \eta$ the vector $1+\eta \in V$ is annihilated by $g_{\alpha}$, and therefore $1+{ }^{c} \in V_{\alpha}^{+}$. From Lemma 4.7 we conclude that $V_{\alpha}^{+}$consists of vectors $c+{ }^{c} \eta, c \in C$. From $C \cap 9=\mathscr{L}$ it then follows that the mapping

$$
\varphi: V_{\alpha}^{+} \rightarrow C / \mathscr{L}, \varphi(c+91)=c+\mathscr{L}
$$

is a $C$-linear isomorphism. Thus $V_{\alpha}^{+} \cong W$ as $C$-modules. Next we observe that $C \cap \mathscr{E}(G) 9 l_{\alpha} \subset \mathscr{L}$, so $9 l_{\alpha} \subset \eta l$ and therefore $V_{\beta}^{+}=A_{\beta, \alpha} V_{\alpha}^{+}=0$ for $\beta<\alpha$. It follows that $[V] \in G_{\alpha}^{\prime}$.

By Lemma 3.5, $\operatorname{rank} K=\operatorname{rank} G$ if and only if $T_{0}=0$.

Theorem 4.9. Let $\operatorname{rank} G=\operatorname{rank} K$. Then for any large weight $\alpha \in A$ the set $G_{\alpha}^{\prime}$ contains exactly one element $[V]$ and $\operatorname{dim} V_{\alpha}^{+}=1$.

Proof. Let $c$ be one of the generators $c_{1}, \ldots, c_{o}$ of $C$ (see Definition 4.1). Then $c \in A_{\alpha}, \operatorname{deg}(c) \leqq n_{c}$ and $[H, c]=0$. Then $c$ can be written in the form described in Lemma 4.5. Since $[H, c]=0, \lambda_{i_{1}}+\lambda_{i_{2}}+\ldots+\lambda_{i_{k}}$ $=0$ for each of the products $u_{i_{1}} u_{i_{2}} \ldots u_{i_{k}}$. Now $\lambda_{i_{1}} \geqq \lambda_{i_{2}} \geqq \ldots \geqq \lambda_{i_{k}}$ and $\lambda_{i_{v}} \neq 0(v=1,2, \ldots, k) \quad\left(T_{0}=0\right)$; thus $\lambda_{i_{k}}<0$ and $u_{i_{1}} u_{i_{2}} \ldots u_{i_{k}}$ $\in 7 m_{\alpha}$. It follows that the generators $c$ belong to the subalgebra $C \cdot \mathbf{1}+$ $C \cap \mathscr{E}(G) 7 m_{\alpha}$ of $C$; hence this is true for all $c \in C$.

We ccnclude that the algebra $C / C \cap \mathscr{E}(G) m_{\alpha}$ is isomorphic (when $\alpha$ is large) to the algebra $C$ of complex numbers and therefore there exists exactly one equivalence class of irreducible (non-zero) $\left.C / C \cap \mathscr{E}(G)^{c}\right) n_{\alpha}$ modules and the dimension of such a module is equal to one. Theorem 4.8 completes the job.

Remark 4.10. The results of this section can be easily extended to the case in which $K$ is a reductive subalgebra of $G$.

University of Jyväskylä

Department of Mathematics

SF - 40100 Jyväskylä 10

Finland 


\section{References}

[1] Harish-Chandra: Representations of a semi-simple Lie group on a Banach space I. - Trans. Amer. Math. Soc. 75, 1953, pp. 185-243.

[2] -》- Representations of semi-simple Lie groups II. - Trans. Amer. Math. Soc. 76, 1954, pp. 26-65.

[3] -》- Representations of semi-simple Lie groups III. - Trans. Amer. Math. Soc. 76,1954 , pp. $234-253$.

[4] Humphreys, J.: Introduction to Lie algebras and representation theory. - Graduate Texts in Mathematics 9, Springer-Verlag, New York - Heidelberg - Berlin, 1972.

[5] Lepowsky, J.: Algebraic results on representations of semi-simple Lie groups. Trans. Amer. Math. Soc. 176, 1973, pp. 1-44.

[6] Lepowskr, J., and G. W. Mc Coldux: On the determination of irreducible modules by restriction to a subalgebra. - Trans. Amer. Nath. Soc. 176, 1973, pp. $45-57$.

[7] Parthasarathy, K. R., R. Ranga Rao and V. S. Varadarajan: Representations of complex semi-simple Lie groups and Lie algebras. - Ann. of Math. 85,1967 , pp. $383-429$.

[8] Warner, G.: Harmonic analysis on semi-simple Lie groups I. - Die Grundlehren der mathematischen Wissenschaften in Einzeldarstellungen 188, SpringerVerlag, Berlin - Heidelberg - New York, 1972. 